\title{
Chimó: tabaco sin humo venezolano asociado a efectos tóxicos bucales y sistémicos. Revisión bibliográfica.
}

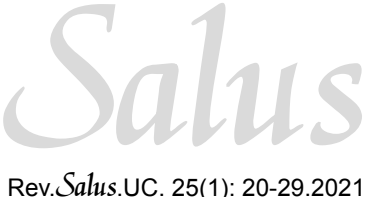

Rev.Salus.UC. 25(1): 20-29.2021

Chimó: Venezuelan smokeless tobacco associated with oral and systemic toxic effects. Bibliographic review.

Miguel Flores Asenso(D) Yalitza Aular (D) Davide Mobili Rocaro (D)

https://doi.org/10.54139/salus.v25i1.6

\section{RESUMEN}

El objetivo de la revisión fue analizar la evidencia científica sobre los efectos tóxicos del consumo del tabaco sin humo (TS) venezolano (chimó) en la cavidad bucal y a nivel sistémico. Fueron empleadas para la búsqueda las bibliotecas virtuales BVS, PubMed, Nature, Scholar Google y LILACS, utilizando la palabra en español: chimó, palabras combinadas: tabaco sin humo, tabaco de mascar, tabaco venezolano, patología bucal, patologías sistémicas; palabras en inglés: smokeless, smoked, tobacco; palabras en portugués: tabaco sem fumaça, se incluyeron artículos libres y artículos completos, tomando como criterios de inclusión y exclusión, que abordaran el tema del chimó y su relación a efectos tóxicos a nivel sistémico y bucal, que no fuesen estudios de prevalencia e incidencia y que hayan sido publicados a partir del año 2000 . Fueron seleccionados un total de 61 (sesenta y uno) artículos en inglés, portugués y español que consideran el TS, incluyendo 14 (catorce) estudios observacionales, descriptivos y explicativos, que valoran los efectos sobre la cavidad bucal y sistémicos del chimo venezolano. Los resultados mostraron que existen pocos estudios con evidencia científica que respalde el papel etiológico del chimó venezolano en los procesos patológicos bucales y sistémicos. No obstante, la existente coincide con los efectos adversos a la salud que son atribuidos al tabaco de combustión, tales como enfermedad cardiovascular, enfermedad pulmonar obstructiva crónica, cáncer, enfermedades metabólicas y a nivel bucal (lesiones pigmentadas en el tejido duro dental y en tejidos blandos, leucoplasias, eritroplasias; desórdenes potencialmente malignos), enfermedad periodontal, cáncer bucal, que están relacionados con estrés oxidativo, lesiones del ADN, lo que lleva a inestabilidad genómica y muerte celular producidos por la nicotina y la cotinina (su principal metabolito). Se requieren más estudios para profundizar en los efectos tóxicos del chimó venezolano en la salud bucal y a nivel sistémico en los consumidores.

Palabras clave: patologías bucales, patologías sistémicas, tabaco de mascar, tabaco sin humo

${ }^{1}$ Maestría en Biología Oral Facultad de Odontología. Universidad de Carabobo.

${ }^{2}$ Maestría en Toxicología Analítica, Centro de Investigaciones Médicas y Biotecnológicas de la Universidad de Carabobo (CIMBUC). Facultad de Ciencias de la Salud

Autor de correspondencia: Miguel Flores Asenso (ID

E-mail: miguelignacioflores@gmail.com

Recibido: 23-10-2020

Aprobado: 30-03-2021
The aim of this review was to analyze the scientific evidence on the toxic effects of the consumption of Venezuelan smokeless tobacco (TS) (chimó) in the oral cavity and at the systemic level. The virtual libraries BVS, PubMed, Nature, Scholar Google and LILACS were used for the search, using the words in Spanish: chimó, combined words: smokeless tobacco, chewing tobacco, Venezuelan tobacco, oral pathology, systemic pathologies; English words: smokeless, smoked, tobacco; words in Portuguese: tobacco sem fumaça, free articles and full articles were included, taking as inclusion and exclusion criteria, addressing the issue of chimó and its relationship to toxic effects at the systemic and oral level, other than prevalence and incidence studies, and those published since year 2000. A total of 61 (sixty-one) articles were selected in English, Portuguese and Spanish that consider ST, including 14 (fourteen) observational, descriptive and explanatory studies, which assess the effects of the Venezuelan chimo both systemic and on the oral and cavity. Results showed that few studies with scientific evidence support the etiological role of the Venezuelan chimó in oral and systemic pathological processes. However, the existing one confirms the adverse effects to the health attributed to combustion tobacco, such as cardiovascular disease, chronic obstructive pulmonary disease, cancer, metabolic diseases, and in the oral cavity (pigmented lesions in dental hard tissue and soft tissues, leukoplakia, erythroplasia; potentially malignant disorders), periodontal disease and oral cancer, which are related to oxidative stress, DNA damage, leading to genomic instability and cell death caused by nicotine and cotinine (its main metabolite). More studies are required to delve into the toxic effects of Venezuelan chimó on oral health and at a systemic level in consumers.

Key words: oral pathologies, systemic pathologies, chewing tobacco, smokeless tobacco

\section{INTRODUCCIÓN}

Las primeras descripciones del uso de la planta de tabaco (Nicotiana tabacum) en Venezuela se relacionan al uso dado por los pueblos indígenas americanos, quienes lo empleaban para inducir estados de conciencia alterados en ritos de iniciación; con fines medicinales y terapéuticos, así como para aliviar el hambre y la sed (1). Sin embargo, la expansión, comercialización y distribución del tabaco a nivel mundial se debe notoriamente al trabajo de la corona española a partir del siglo XV (2). Actualmente, el mercadeo mundial del tabaco ocupa una amplia gama de productos que incluyen al tabaco de combustión, o tabaco inhalado, o tabaco con humo, entre el que está el cigarro, habanos, pipa, y de reciente data los dispositivos vaporizadores; y las 
otras formas de tabaco en pasta, polvo, disolventes, gomas de mascar, conocidas como tabaco sin humo (TS), o tabaco sin combustión, el cual se consume masticado, succionado, desleído o inhalado por vía nasal (3).

Los productos de TS son un grupo diverso cuyo componente principal es el tabaco, manufacturado a concentraciones variables e ingredientes adicionales (para aromatizar, alcalinizar y saborizar), cuya composición y proporciones se modifican de acuerdo con la idiosincrasia de la región; siendo algunos de estos ingredientes sustancias consideradas altamente tóxicas y carcinogénicas (4). Entre los productos de TS está el chimó.

El chimó, también conocido como tabaco de mascar venezolano, es un extracto resultante de la maduración y curación de las hojas del tabaco ( $\mathrm{N}$. tabacum o $\mathrm{N}$ rustica) (5); que posteriormente son hervidas durante varias horas o días; el resultado es un producto concentrado, una pasta pegajosa, pesada, de coloración negra, que puede añejarse hasta 2 años, en contenedores como taparas (frutos secos del árbol Tapara) o envuelto en hojas de plátano. La pasta madurada es sazonada con otros ingredientes, tales como bicarbonato de sodio (alcalinizante), azúcar moreno, cenizas del árbol de mamón (Meliccoca bijuga), vainilla, anís dulce, entre otros, que varían según la región (urea, sal de Urao, hipoclorito de sodio al 10\%), y luego son envasados en latas pequeñas (6-7).

Entre los componentes principales del chimó están los alcaloides $(0,5-5.0 \%)$, que contienen la nicotina como compuesto predominante $(85-95 \%$ de los alcaloides totales), terpenos $(0.1-3,0 \%)$, polifenoles $(0.5-4.5 \%)$, fitoesteroles $(0.1-2 \%)$, nitratos alcalinos $(0,01-5 \%)$ y al menos 30 compuestos metálicos (8,9). Además de 23 nitrosaminas específicas de tabaco (TSNA) y 28 pesticidas (10), resultantes de la reducción del nitrato en nitrito, proceso llevado a cabo por una serie de bacterias y hongos (11-14).

Se debe señalar que durante el proceso de fabricación pueden ocurrir cambios esenciales en la composición química de las hojas de tabaco, debido a su exposición a medios contaminados, durante las diversas etapas (cultivo, curado y almacenamiento) (11-14); como el polonio-210, elemento radiactivo presente en el fertilizante de tabaco); además del procesamiento (fermentación-añejamiento) lo que conduce a la presencia, entre sus componentes de una gran variedad de metales, metaloides, hidrocarburos policíclicos aromáticos (15), y algunos modificadores alcalinos propios del chimó, que elevan el $\mathrm{pH}$, clasificados por la International Agency for Research on Cancer (IARC) (2007) como carcinógenos del Grupo 2 (probablemente carcinógeno para los seres humanos) (16). Todos nocivos a la salud (17).

La nicotina (principal constituyente del chimo) tiene una vida media plasmática muy corta (aproximadamente 30 minutos) (18) y la mayor parte de la nicotina $(70 \%$ al $80 \%)$ es rápidamente metabolizada en el hígado a través de las enzimas del citocromo P450, principalmente CYP2A6 y convertida en su principal metabolito, la cotinina, un alcaloide considerado tóxico, que tiene una vida media biológica más larga (10 a 30 horas) y también es capaz producir los mismos efectos que la nicotina, probablemente debido a la similitud estructural (19),

Además, la nicotina es un precursor importante de dos TSNA presentes en el tabaco sin humo, la N'-nitrosonornicotina (NNN) y 4- (metilnitrosamino) -1- (3-piridil) -1-butanona (NNK), clasificadas por la IARC (2007) como carcinógenos del Grupo 1 (existe suficiente evidencia de carcinogenicidad en humanos) (20).

En Venezuela, según el Global Youth Tobacco Surveys 2007-2010 (GYTS venezolano), primer sistema de vigilancia poblacional específico del tabaco para estimar la prevalencia del uso de productos de TS y comportamientos relacionados en el país, realizado entre estudiantes de los grados 7-9, mostró que a nivel nacional y para los estados Barinas, Cojedes, Monagas, Nueva Esparta, Trujillo, Zulia, Yaracuy y Lara en los años 2000, 2004 y 2008 la prevalencia del uso de chimó no fue uniforme entre los estados: Varió del $3,8 \%$ al $20,7 \%$ para los niños y del $2,0 \%$ al $6,6 \%$ para las niñas, con una mayor prevalencia general en Barinas, Cojedes, Monagas y Lara (21). El GYTS también encontró que los estudiantes de séptimo grado usaban chimó con más frecuencia que cigarrillos, que puede reflejar el hecho de que los programas escolares de prevención del tabaquismo solo abordan productos de tabaco con humo (21-23).

La Encuesta Estatal de Salud Cardíaca de Lara de 2008 notifica que adultos mayores de 15 años, el 15,4\% de los hombres y el $3,1 \%$ de las mujeres, han consumido chimó alguna vez, mientras que el $6,2 \%$ de los hombres y el $1,5 \%$ de las mujeres son consumidores actuales (24).

El consumo de chimó, y el de otros productos de TS han sido relacionados directamente con la aparición y avance de muchas enfermedades. En este sentido se han asociado a desórdenes potencialmente malignos de la cavidad bucal, como leucoplasia, eritroplasia y liquen plano; además se han señalado como causa de cáncer de la orofaringe, así como otras condiciones como caries dental y enfermedad periodontal (25).

En consecuencia, el tabaquismo es considerado como un problema de salud pública a nivel mundial. La Organización Mundial de la Salud (OMS) (26-30) reporta que cada año, más de 8 millones de personas fallecen a causa del tabaco y lo describe como una epidemia, en cualquiera de sus presentaciones, sea para fumar, inhalar o masticar. No obstante, a pesar de que existen campañas y leyes que regulan el consumo de tabaco, estas han centrado la mayoría de las acciones al tabaco inhalado, siendo una realidad que en países como Venezuela y otras regiones de Sudamérica 
se ha incrementado el consumo de productos de TS, principalmente el chimó constituyéndose en un hábito con una influencia social y cultural que ha trascendido fronteras, al que le han atribuido propiedades preventivas y curativas en enfermedades, así como otras indicaciones. Aun cuando los supuestos beneficios atribuidos al chimó no tienen un soporte científico que validen sus posibles propiedades (31).

Adicionalmente, en el país los estudios sobre sus efectos en la salud bucal y sistémica son escasos y la población no está suficientemente educada acerca de los riesgos de su consumo. Sobre la base de estos planteamientos se realizó la presente revisión con el objetivo de analizar la evidencia científica sobre los efectos tóxicos del consumo del chimó venezolano en la cavidad bucal y a nivel sistémico.

\section{MATERIALES Y MÉTODOS}

Fueron empleadas las bibliotecas virtuales BVS, PubMed, Nature, Scholar Google y LILACS, se utilizaron para la búsqueda palabras en español: chimó, palabras combinadas: tabaco sin humo, tabaco de mascar, tabaco venezolano, patología bucal, patologías sistémicas; palabras en inglés: smokeless, smoked, tobacco; palabras en portugués: tabaco sem fumaça, utilizando el esquema de Rostron et al. (32) ajustado a los requerimientos del presente estudio. Se incluyeron artículos libres y artículos completos, se tomó como criterios de inclusión y exclusión, que abordaron el tema del chimó venezolano y su relación a efectos tóxicos a nivel sistémico y bucal, que no fuesen estudios de prevalencia e incidencia y que hayan sido publicados a partir del año 2000.

De esta manera, se seleccionaron un total de 61 (sesenta y uno) artículos en inglés, portugués y español, de igual forma, fueron incluidos en la revisión 14 (catorce) estudios observacionales, descriptivos y explicativos (Figura 1), que valoran los efectos sobre la cavidad bucal y sistémicos. Una vez seleccionados los artículos se procedió a extraer la información más relevante, analizarla y compararla para su presentación y discusión.

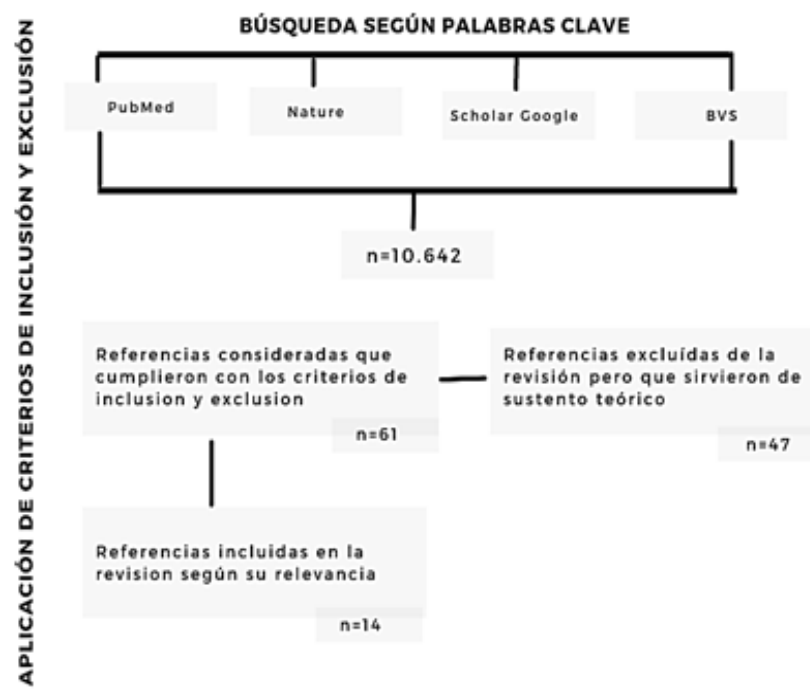

Figura 1. Esquema de metodología adaptada de Rostron et al. (32)

\section{RESULTADOS}

En la Tabla 1 se muestran los 14 estudios sobre los efectos tóxicos del chimo venezolano realizados en el país, 5 (cinco) investigaciones in vitro, 3 (tres) en modelos de experimentación animal y 6 (seis) en humanos. En la cual se observan las variables utilizadas, tipos de estudio, efectos tóxicos estudiados y el o los autores y el año de publicación del estudio.

Tabla 1. Estudios sobre los efectos tóxicos del Chimó venezolano.

\begin{tabular}{|c|c|c|c|c|c|}
\hline \multirow[b]{2}{*}{ Variable del Estudio } & \multirow[b]{2}{*}{ In vitro } & \multicolumn{2}{|c|}{ Tipo de estudio } & \multirow[b]{2}{*}{ Efecto tóxico } & \multirow[b]{2}{*}{ Autor /año } \\
\hline & & $\begin{array}{l}\text { Experimental } \\
\text { en animales }\end{array}$ & $\begin{array}{l}\text { Biomonitoreo } \\
\text { en humanos }\end{array}$ & & \\
\hline Cambios histológicos & & * & & $\begin{array}{ll}\text { - } & \text { Ortoqueratosis. (33) } \\
\text { - } & \text { Displasia epitelial leve, moderada. (33) } \\
\text { - } & \text { Hiperqueratosis. (33) } \\
\text { - } & \text { Acantosis. (33) } \\
\text { - } & \text { Fibrosis y colagenización del estroma. (33) } \\
\text { - } & \text { Alteraciones en arquitectura del epitelio. (33) }\end{array}$ & $\begin{array}{l}\text { Sosa et al. } 2009 \\
\text { (33) }\end{array}$ \\
\hline Cambios clínicos & & * & & $\begin{array}{ll}\text { - } & \text { Agradamiento gingival. (34) } \\
\text { - } & \text { Cambio de consistencia de encía. (34) } \\
\text { - } & \text { isquémicas. (34) } \\
\text { - } & \text { Incremento de queratinización. (34) } \\
\text { - } & \text { Migras periodontales. (34) } \\
\text { - } & \text { Leucoplasias y eritroplasias. (34) } \\
\text { - } & \text { alteraciones en la microarquitectura cardiaca. (35) } \\
& \text { nerfil isquémico: baja frecuencia cardiaca y altos } \\
& \text { de subunidad CK-MB. (35) }\end{array}$ & $\begin{array}{l}\text { Rosales et al. } \\
2008 \text { (34) }\end{array}$ \\
\hline
\end{tabular}




\begin{tabular}{|c|c|c|c|c|c|}
\hline \multirow[b]{2}{*}{ Variable del Estudio } & \multirow[b]{2}{*}{ In vitro } & \multicolumn{2}{|c|}{ Tipo de estudio } & \multirow[b]{2}{*}{ Efecto tóxico } & \multirow[b]{2}{*}{ Autor /año } \\
\hline & & $\begin{array}{l}\text { Experimental } \\
\text { en animales }\end{array}$ & $\begin{array}{l}\text { Biomonitoreo } \\
\text { en humanos }\end{array}$ & & \\
\hline Cardiotoxicidad & & * & & $\begin{array}{l}\text { - } \quad \text { Alteraciones en la microarquitectura cardiaca. (35) } \\
\text { Perfil isquémico: baja frecuencia cardiaca y altos } \\
\text { niveles de Creatinina Quinasa (CK) y su Isoenzima } \\
\text { de subunidad CK-MB. (35) }\end{array}$ & $\begin{array}{l}\text { Álvarez et al. } \\
2016(35)\end{array}$ \\
\hline $\begin{array}{l}\text { Potencial mutagénico de } \\
\text { los extractos de tabaco } \\
\text { de mascar mediante la } \\
\text { Prueba de Ames. }\end{array}$ & * & & & $\begin{array}{l}\text { Baja mutagenicidad } \\
\text { (Test de Ames con o sin S9) (6). }\end{array}$ & $\begin{array}{l}\text { Jarpa et al. } \\
2003(6)\end{array}$ \\
\hline $\begin{array}{l}\text { Erosión dental asociada } \\
\text { a exposición al chimó }\end{array}$ & * & & & $\begin{array}{l}\text { Estructura adamantina erosionada. (36) } \\
\text { - } \quad \text { Superfión de moderada a completa. (36) } \\
\text { depresiones en la superficie y detritus. (36) } \\
\text { - Cambio de coloración de esmalte. (36) }\end{array}$ & $\begin{array}{l}\text { Febres et al. } \\
2017(36)\end{array}$ \\
\hline pH y adicción & * & & & $\begin{array}{l}\text { Los valores de } \mathrm{pH} \text { de muestras de chimo } \\
\text { venezolano mostraron un promedio de } 9.3 \pm 0.6 \text {, } \\
\text { significativamente }(\mathrm{p}<0.05) \text { más alto de } \mathrm{pH} \text { que } \\
\text { muestras de otros TS. A pH altos la nicotina libre } \\
\text { presenta mayor absorción, atraviesa barreras } \\
\text { biológicas, favoreciendo la adicción y los efectos } \\
\text { tóxicos en general (37) }\end{array}$ & Jarpa 2003 (37) \\
\hline
\end{tabular}

\begin{tabular}{|c|c|c|c|c|c|}
\hline Niveles de nicotina & * & & • & $\begin{array}{l}\text { Los niveles de nicotina en las muestras de chimó } \\
\text { venezolano están en el rango de } 0,009 \mathrm{mg} / \mathrm{g} \text { y } \\
10,8 \mathrm{mg} / \mathrm{g} \text {. Los niveles más bajos son para chimo } \\
\text { venezolano artesanal, y los más altos para chimo } \\
\text { venezolano industrializado. (8) }\end{array}$ & $\begin{array}{l}\text { Corredor- } \\
\text { Aranguren et al } \\
2013(8)\end{array}$ \\
\hline Calidad microbiológica & * & & - & Aumento de carga fúngica (38) & $\begin{array}{l}\text { Millán-Mendoza } \\
\text { et al. } 2016(38)\end{array}$ \\
\hline $\begin{array}{l}\text { Lesiones en la mucosa } \\
\text { bucal }\end{array}$ & & * & $\begin{array}{l}\cdot \\
. \\
. \\
. \\
. \\
.\end{array}$ & $\begin{array}{l}\text { Lesiones leucoplásicas exofílicas de textura } \\
\text { rugosa. (39) } \\
\text { Leucoeritroplasias. (39) } \\
\text { Inflamación generalizada de la mucosa. (39) } \\
\text { Hemorragias. (39) } \\
\text { Leucoplasia verrugosa. (39) } \\
\text { Displasias leves y severas. (39) } \\
\text { Carcinoma de células escamosas. (39) }\end{array}$ & $\begin{array}{l}\text { Bermúdez. } \\
2011(39)\end{array}$ \\
\hline Cambios celulares & & * & •. & $\begin{array}{l}\text { Infiltrado inflamatorio. (40) } \\
\text { Pérdida de la relación núcleo - citoplasma. (40) } \\
\text { Presencia de binucleaciones y halos perinucleares. } \\
(40)\end{array}$ & $\begin{array}{l}\text { Parra et al. } \\
2014(40)\end{array}$ \\
\hline Flora bacteriana & & * & - & $\begin{array}{l}\text { Aumento de carga bacteriana acidógena en } \\
\text { cavidad bucal }(41)\end{array}$ & $\begin{array}{l}\text { Sosa et al. } \\
2008(41)\end{array}$ \\
\hline $\mathrm{pH}$ y flujo salival & & * & - & Modificación del pH salival y flujo salival (42) & $\begin{array}{l}\text { Sánchez et al. } \\
2009(42)\end{array}$ \\
\hline $\begin{array}{l}\text { Diabetes mellitus tipo } 2 \\
\text { (DM2) }\end{array}$ & & * & • & $\begin{array}{l}\text { El consumo de Chimó aumenta }(77 \%) \text { las } \\
\text { probabilidades de padecer DM2 }(43) \text {. }\end{array}$ & $\begin{array}{l}\text { González-Rivas } \\
\text { et al. } 2017 \text { (43) }\end{array}$ \\
\hline $\begin{array}{l}\text { Hipertensión Arterial } \\
\text { Sistémica / DM2 }\end{array}$ & & * & - & $\begin{array}{l}\text { Los valores de HAS fueron más bajos en los } \\
\text { consumidores de chimó con DM2 y sin DM2 (44). }\end{array}$ & $\begin{array}{l}\text { González-Rivas } \\
\text { et al. } 2017 \text { (44) }\end{array}$ \\
\hline
\end{tabular}

\section{Salus}




\section{DISCUSIÓN}

En la revisión realizada se observó que los efectos tóxicos de los productos de TS incluyendo el chimó, se han relacionado con la nicotina, cuya biodisponibilidad se incrementa con niveles altos de $\mathrm{pH}$. En este sentido, estudios han mostrado valores de $\mathrm{pH}$ mayores de 9 en diversas marcas de chimó venezolano, lo cual favorecería una mayor biodisponibilidad de nicotina, que incluye a la nicotina libre (también llamada no ionizada) y nicotina ionizada (45), conduciendo a una mayor absorción y permanencia en los tejidos, favoreciendo los efectos citotóxicos de chimó (37).

Los efectos del chimó han sido clasificados a nivel clínico como eventos a corto, mediano y largo plazo. A corto plazo los cambios están directamente relacionados con la absorción de la nicotina libre, no ionizada, que es la forma que atraviesa las membranas celulares, a través de la mucosa bucal, esta sustancia posee características vasoconstrictoras lo que provoca un aumento de la presión sanguínea; razón por lo cual este hábito también se ha relacionado con alteraciones cardiovasculares $(6,46)$.

A mediano plazo los efectos del chimó se ven reflejados a nivel de la cavidad bucal, donde pueden observarse recesiones gingivales, manchas dentales, así mismo algunas estructuras de la mucosa presentas zonas rugosas y blanquecinas sobre todo en el área donde se tiende a colocar la porción de pasta, estas lesiones corresponden a leucoplasias y/o queratosis que aparecen al cabo de 2 años de consumo $(6,34,39,40)$.

A largo plazo, además de las lesiones provocadas durante la absorción local, en pacientes con consumo de TS por tiempo prolongado, aproximadamente 8,1 años de consumo (6), se han reportado lesiones neoplásicas a nivel de la cavidad oral, faringe, laringe, acompañado también por el desarrollo de carcinoma espinocelular (47-48).

Se ha descrito que para su consumo el chimo es colocado directamente en la boca, entre el labio o la mejilla y la encía, se deja allí durante un tiempo, generalmente $30 \mathrm{~min}$, aumentando el contacto del chimó con la mucosa bucal (31) lo que se ha relacionado con la aparición de leucoplasias y cáncer en mejillas y encías (48), en un $50 \%$ de los usuarios (49).

En relación con los efectos tóxicos del Chimó sobre mucosa y microambiente bucal, la revisión realizada mostró que los productos de TS son considerados carcinógenos y su consumo se relaciona a cáncer bucal (25).

Al chimó se le considera un producto tabáquico con potencial mutagénico. Jarpa en 2003 (6), realizó un estudio in vitro para analizar el potencial mutagénico del chimó, utilizando la Prueba de Ames. Fueron analizadas cuatro marcas de tabaco de mascar (dos productos norteamericanos: Copenhagen $₫$, y Skoal Bandits $₫$, uno de la India: Maythi $\circledR$ y uno venezolano marca El Tigrito $\left.{ }^{\circledR}\right)$. Los resultados evidenciaron baja mutagenicidad, pero el mismo autor señaló que para considerarlos como no mutagénicos se requiere mayores estudios y análisis químico más precisos.

En un estudio realizado por Rosales et al. (34) (Tabla1) se evaluó los cambios clínicos que puede ocasionar el chimó en los tejidos periodontales de 22 ratas macho Wistar. Los resultados mostraron agradamiento gingival que modificó la consistencia y redujo en un $90 \%$ ell puntillado de la encía, zonas isquémicas debido al incremento de la queratinización $(100 \%)$ evidenciadas por una coloración blanca y; entre las semanas 16 y 20 presencia de sacos periodontales (36\%), migraciones gingivales apicales (63\%), leucoplasias y eritroplasias $(28 \%)$, sugiriendo que estos cambios clínicos en la mucosa bucal están directamente relacionados con el tiempo de exposición al chimó y la forma de consumo que aumenta el contacto del chimó con la mucosa bucal (31).

Otros autores han evidenciado que los cambios clínicos ocasionados por el consumo de tabaco son producidos por la nicotina y la cotinina (su principal metabolito) que inducen un efecto citotóxico (18). Aguiar et al. (50) han señalado que la acción de la cotinina y la nicotina está relacionada con el estrés oxidativo, que puede resultar en lesiones del ADN, lo que lleva a inestabilidad genómica y muerte celular. La guanina se considera la base más susceptible a la oxidación debido al bajo potencial redox, siendo el 8-oxo-7,8-dihidro2'-desoxiguanosina (8-oxodG) el daño más común. Asimismo, se ha demostrado un aumento de la catalasa (CAT), responsable de la descomposición del peróxido de hidrógeno en agua y oxígeno, en moluscos de la especie del género Helix expuestos a la nicotina (51), otros autores han demostrado que la nicotina afecta la acción del superóxido dismutasa, catalasa y glutatión reductasa, lo que indica un mecanismo de estrés oxidativo en el daño del ADN causado por la nicotina (52).

Sosa et al. (33) (Tabla 1), han señalado que la citotoxicidad puede expresarse en alteraciones histológicas y utilizando un diseño experimental similar a Rosales et al. (34), observó que el grupo experimental presentó ortoqueratosis (54 \%), displasia epitelial leve (45\%), granulocitosis, hiperqueratosis, acantosis, fibrosis y colagenización del estroma, e inflamación crónica en un $19 \%$ y displasia moderada $10 \%$, sin cambios aparentes en el grupo control, evidenciando la asociación entre consumo de chimó, tiempo de consumo y citotoxicidad.

Sugieren que estas manifestaciones histológicas que el chimó induce cambios en los tejidos bucales que inician en procesos inflamatorios, aumento de la producción 
de queratina y colagenización del estroma, desordenes celulares y alteraciones de la arquitectura del epitelio, llegando a ser lesiones irreversibles, indicando que las alteraciones clínicas son consecuencia de modificaciones a nivel celular, los componentes del tabaco en sus distintas formas producen cambios en cuanto a calidad de los organelos, al aumento o disminución del número de células y variación en su forma.

En humanos, Bermúdez (39) (Tabla 1), en un caso clínico, observó la aparición de lesiones potencialmente malignas que evolucionan a cáncer en la mucosa bucal relacionadas al consumo de chimó, desde la niñez, en un paciente de 78 años, mostrando inicialmente lesiones leucoplásicas en el reborde residual en mandíbula, de textura rugosa exofílica y a nivel de piso de boca presentó leucoeritroplasias, inflamación generalizada con dolor y hemorragia; extrabucalmente se evidenciaba queilitis angular severa que ocasionaba dificultad para la apertura bucal, el diagnóstico histopatológico mostró leucoplasia verrugosa con cambios displásicos, leves y severos, en zona edéntula mandibular y en piso de boca que evolucionó en tres meses a carcinoma de células escamosas, coincidiendo con Van der Waal (53) que sostiene que el consumo de tabaco no inhalado es un agente etiológico de alta agresividad en lesiones potencialmente malignas que pueden evolucionar a cáncer principalmente de la zona de cabeza y cuello.

Al igual que en los modelos animales de los estudios de Rosales et al. (34) y Sosa et al.(33) Parra et al. (40) (Tabla 1) observaron la presencia de infiltrado inflamatorio, pérdida de la relación núcleo - citoplasma, presencia de binucleaciones y halos perinucleares en consumidores de chimo, estos hallazgos están en concordancia con las alteraciones clínicas de la zona donde se aplicó el cito cepillo, estableciéndose una relación directa y estadísticamente significativa entre la cantidad de chimó consumida diariamente y los cambios celulares observados.

El consumo de chimo se ha asociado a erosión y caries dental. Febres et al. (36) (Tabla 1) estudiaron in vitro la erosión dental asociada a la exposición al chimó, y los resultados clínicos mostraron erosión de moderada a completa en el centro de las estructura adamantina, manifestando irregularidades, depresiones en la superficie y detritus, concluyendo que el chimó produce pérdida de la superficie del esmalte tanto clínica como ultraestructuralmente, con presencia de erosión de moderada a completa coincidiendo con Muthukrishnan et al. (25) que ha señalado que el alto contenido de agentes edulcorantes y aromatizantes en los productos de tabaco sin humo son los responsable de la caries dental.

Otro aspecto encontrado en la revisión son los efectos del chimó en la prevención de la formación de biopelícula y actividad antibacteriana. Sosa et al. (41) (Tabla 1). Compararon la flora bacteriana que se adosa a las superficies supragingivales de los dientes en individuos consumidores y no consumidores de chimó. Para los individuos consumidores de chimó la medición del índice O'Leary, que indica el porcentaje de biopelícula en la superficie dental y permite evaluar el grado de higiene bucal, obtuvo los valores más elevados del conjunto examinado, igualdad del morfotipo bacteriano aislado, con predominio de cocos grampositivos aeróbicos y microaerófilos en ambos grupos, además la carga bacteriana acidógena fue superior, sugiriendo que el chimó no ejerce efecto inhibitorio sobre las cargas bacterianas evaluadas y los consumidores presentan mayor predisposición a desarrollar patologías asociadas a la formación de biopelículas dentales. MillánMendoza et al. (38) determinaron la calidad microbiológica de cuatro marcas comerciales de chimó de uso frecuente en la población del estado Mérida. En las muestras analizadas no se observó crecimiento bacteriano, pero si abundante crecimiento fúngico de los géneros Rhizopus, Aspergillus, Rhodotorula, Fusarium, Penicillium, Helminthosporium, y Hormodendrum y aunque estos géneros no se encuentran habitualmente en el humano, pueden causar micosis oportunistas, especialmente en los consumidores inmunocomprometidos, ocasionando graves daños a su salud.

Los efectos de la nicotina sobre el SNC se relacionan, por una parte, con una acción en sitios específicos de los receptores colinérgicos nicotínicos $(\mathrm{nAChR})$ en la presinapsis y estimula la liberación de varios neurotransmisores como la dopamina y la noradrenalina, que juegan un papel importante en la adicción a la nicotina y en el síndrome de abstinencia respectivamente (54), Por la otra parte, con la estimulación del centro nervioso encargado de la estimulación de los núcleos salivales del Bulbo raquídeo (55) En un primer momento incrementa el flujo salival y posteriormente disminuye considerablemente $(56,57)$, lo cual está en concordancia con estudios que sostienen que no hay diferencia en el flujo salival entre consumidores y no consumidores de tabaco (58).

En contraste, Sánchez et al. (42) (Tabla 1) en un estudio donde evaluaron los efectos del chimó sobre el flujo salival y el pH y sus consecuencias sobre la salud bucal, los individuos consumidores de chimó evidenciaron un promedio de flujo salival de $1,22 \mathrm{~mL} / \mathrm{min}$ y un $\mathrm{pH}$ salival de 6,9 en consumidores de chimo, en comparación con un flujo salival de $0,66 \mathrm{~mL} / \mathrm{min}$ y un $\mathrm{pH}$ de 7,05 para los no consumidores. Hubo un $84,85 \%$ de incremento del volumen del flujo salival a expensas de los consumidores de chimó, coincidiendo con Rooban et al. (59), que sostienen que el flujo salival y el pH están alterados en los consumidores de TS, lo que hace que la mucosa bucal sea más vulnerable a 
los efectos tóxicos de sus componentes. No obstante, no hay consenso científico ante este indicador.

En cuanto a los efectos del chimó a nivel sistémico se ha descrito que el consumo de TS se encuentra relacionado con factores de riesgo cardíaco, como elevada presión sanguínea, diabetes, e hiperlipidemias, así como una alta tasa de mortalidad por alteraciones cardiovasculares $(32,46)$.

La nicotina está relacionada con una elevación significativa de los niveles plasmáticos de varias hormonas, la hormona adenocorticotrópica, cortisol, la hormona de crecimiento, prolactina y vasopresina, favoreciendo la liberación de noradrenalina y adrenalina, que produce un aumento de ácidos grasos en sangre, ocasionando efectos cardiovasculares, tales como el incremento significativo de la presión arterial sistólica y diastólica y de la frecuencia cardíaca $(45,46)$,

El aumento de células endoteliales: leucocitos, plaquetas, fibrinógeno, hematocrito, la formación de trombos y placas de ateromas, responsables de los eventos coronarios, del proceso arteriosclerótico (32) y de procesos proinflamatorios que modifican la estructural epitelial, que se evidencia en atrofia celular, hiperplasia, metaplasia y displasia que pueden evolucionar a lesiones neoplásicas $(39,40)$.

Existen evidencias de que la nicotina afecta la acción del superóxido dismutasa, catalasa y glutatión reductasa (52), lo que indica un mecanismo de estrés oxidativo en el daño del ADN y que el proceso de oxidación es endógeno y causa un daño considerable al ADN.

El desequilibrio entre la formación y eliminación de ROS puede contribuir a enfermedades degenerativas como diabetes, cáncer y envejecimiento en general. Estas especies altamente reactivas pueden formar una amplia variedad de aductos de ADN mutagénicos (60). Los ataques de ADN por ROS pueden causar la formación de 8-oxodG, que se forma por la hidroxilación de desoxiguanosina en el ADN (49). La exposición crónica a la nicotina puede aumentar la producción de ROS en Helix aspersa con posible daño al ADN y desregulación del ciclo celular, así como un aumento del óxido nítrico (NO) que puede desempeñar un papel en la genotoxicidad de la nicotina (50). Feltes et al. (61) utilizando sistemas biológicos demostraron que la nicotina puede estar relacionada con el desequilibrio oxidativo de la célula y afectar la proliferación celular.

Respecto a la diabetes, González-Rivas et al. (43) asociaron el consumo de chimó a diabetes mellitus tipo 2 (DM2). El estudio mostró que la frecuencia de DM2 fue significativamente mayor en consumidores de chimó que en
Ios no consumidores. Así, el consumo de Chimó aumenta en un $77 \%$ las probabilidades de padecer DM2 (Tabla 1). Sin embargo, estos mismos autores González-Rivas et al. (44) (Tabla 1) en otro estudio, vinculan el consumo de chimó con valores más bajos de Hipertensión Arterial Sistémica (HSA), frecuencia cardíaca e índice de masa corporal (IMC) en individuos con DM2 mayores de 50 años, sin otros datos sobre esta asociación contradictoria.

En contraste, estudios realizados en un modelo larvario de pez cebra Danio rerio, utilizando un extracto acuoso de chimó en el que se identificaron compuestos como la nicotina, derivados de esteroles, cotinina y 2-fluorofenilhidrazina; los efectos tóxicos predominantes fueron desorganización de la arquitectura del tejido cardiaco, vacuolización del tejido de miocardio y endocardio, reducción significativa de la frecuencia cardiaca e incremento de las enzimas creatinina quinasa (CK) y su sub unidad CK-MB; todas variables que definen una disfunción y compromiso morfo-funcional cardiaco (35).

La sustentación científica referida a efectos cardiovasculares ocasionados por el consumo de chimó venezolano no es concluyente en establecer una vinculación proporcional entre consumo y efectos tóxicos, por lo cual se hace necesario mayores estudios.

\section{CONCLUSIONES}

En la presente investigación se llevó a cabo una revisión de la literatura donde se incluyeron 61 (sesenta y un) artículos libres y completos, de los cuales 14 (catorce) abordaron el tema del consumo de chimó venezolano y sus efectos tóxicos a nivel sistémico y bucal, en los cuales se observó que los efectos están relacionados con el estrés oxidativo, que puede resultar en lesiones del ADN, lo que lleva a inestabilidad genómica y muerte celular, producido por la nicotina y su principal metabolito la cotinina, además las TSNA y otros componentes del tabaco, han sido calificados por la IARC como carcinógenos tipos 1 y 2 en humanos. No obstante, las evidencias científicas del consumo de chimó venezolano sobre la toxicidad para la salud humana, en particular, sobre las desordenes potencialmente malignos que pueden evolucionar a cáncer bucal y ser el agente etiológico de enfermedades crónicas sistémicas, metabólicas y obstructiva, no son suficientes y algunos contradictorios, por lo tanto se hace necesario incrementar las investigaciones al respecto que proporcionen datos que contribuyan a esclarecer su riesgo sobre la salud así como promover y diseñar campañas educativas sobre el consumo de las diversas formas de TS y específicamente del chimó, además de la regulación de su comercialización, más aún cuando el producto ha trascendido las fronteras del país debido a la migración de venezolanos en el mundo.

\section{Salus}




\section{REFERENCIAS BIBLIOGRÁFICAS}

1. Brandt, C. Historia del tabaco en Venezuela, 2019. Revista Latino Aficionado Disponible en https://latinoaficionado.com. $\mathrm{mx} / 2019 / 12 / 15 /$ historia-tabaco venezuela/\#: :text=Las $\% 20$ primeras $\% 20$ descripciones $\% 20$ de $\% 20$ la,y\%20la $\% 20$ sed $\% 20$ $\% 5 B 1 \% 5 D$ [Consulta el 15 de febrero de 2021]

2. Monteverde HR, Magaña AR. Breves comentarios sobre la historia del tabaco y el tabaquismo. Rev Inst Nal Enf Resp Mex. 2006;19(4):297-300.

3. WORLD HEALTH ORGANIZATION. IARC Monographs on the Evaluation of Carcinogenic Risks to Humans. VOLUME 89 Smokeless Tobacco and Some Tobacco-specific N-Nitrosamines, pag.33-34. WORLD HEALTHORGANIZATION, INTERNATIONAL AGENCY FOR RESEARCH ON CANCER, 2007. [consulta el 03 de marzo de 2021].

4. WORLD HEALTH ORGANIZATION. IARC Monographs on the Evaluation of Carcinogenic Risks to Humans. VOLUME 89 Smokeless Tobacco and Some Tobacco-specific N-Nitrosamines, pag.48-55. WORLD HEALTHORGANIZATION, INTERNATIONAL AGENCY FOR RESEARCH ON CANCER, 2007. [consulta el 03 de marzo de 2021].

5. National Cancer Institute and Centers for Disease Control and Prevention. Smokeless Tobacco and Public Health: A Global Perspective. Pag 286. Bethesda, MD: U.S. Department of Health and Human Services, Centers for Disease Control and Prevention and National Institutes of Health, National Cancer Institute. 2014; NIH Publication No. 14-7983. Disponible en: https://cancercontrol.cancer.gov/brp/tcrb/smokeless-tobacco. (Acceso 16 de marzo de 2020)

6. Jarpa P. Potencial mutagénico del tabaco de mascar venezolano. Revista de la facultad de farmacia. 2003; 45 (2):26.

7. Granero R, Jarpa P. Uso de chimo entre adolescentes en Venezuela. Encuesta mundial sobre tabaquismo en jóvenes 1999-2008. Acta Odontológica Venezolana,2008; 49(3): 1-9.

8. Corredor-Aranguren A, Chidiak-Tawil S, Jarpa-Remaggi $P$, Urdaneta-Paredes L, Sánchez-Contreras N, AparicioZambrano R, et al. Determinación del nivel de nicotina en el chimó venezolano. Rev Fac Farm. 2013; 55 (2): 6-10.

9. National Cancer Institute and Centers for Disease Control and Prevention. Smokeless Tobacco and Public Health: A Global Perspective. Pag 288. Bethesda, MD: U.S. Department of Health and Human Services, Centers for Disease Control and Prevention and National Institutes of Health, National Cancer Institute. 2014; NIH Publication No. 14-7983. Disponible en: https://cancercontrol.cancer.gov/brp/tcrb/smokeless-tobacco. (Acceso 16 de marzo de 2020)

10. WORLD HEALTH ORGANIZATION. IARC Monographs on the Evaluation of Carcinogenic Risks to Humans. VOLUME 89 Smokeless Tobacco and Some Tobacco-specific N-Nitrosamines, pag.55. WORLD HEALTH ORGANIZATION, INTERNATIONAL AGENCY FOR RESEARCH ON CANCER, 2007. [consulta el 03 de marzo de 2021].

11. García-Rubido M, León D, Acosta-Aguiar Y, Cruz-Hernández Y. Influencia de Canavalia ensiformis micorrizada en algunas variables morfológicas y de rendimiento del tabaco negro cultivado al sol. Cultivos Tropicales. 2017; 38(2):22-23.
12. Olivo J, Capcha J, Chávez J. Bacterias y hongos en el humo aspirado de cigarrillo. Revista ECI. 2016 diciembre; 13 (2):26.

13. González L, Jiménez M, Vaquero L, Paz I, Falcón A, Araujo L. Evaluación de la aplicación de quitosana sobre plántulas de tabaco (Nicotiana tabacum). Centro Agrícola. 2017;44(1): 34 40.

14. Ludwig M, Wilmes $P$, Schrader $S$. Measuring soil sustainability via soil resilience. Science of The Total Environment. Elsevier. 2017 out; $615(1): 1-10$

15. WORLD HEALTH ORGANIZATION. IARC Monographs on the Evaluation of Carcinogenic Risks to Humans. VOLUME 89 Smokeless Tobacco and Some Tobacco-specific N-Nitrosamines, pag.57. WORLD HEALTH ORGANIZATION, INTERNATIONAL AGENCY FOR RESEARCH ON CANCER, 2007. [consulta el 03 de marzo de 2021].

16. Stanfill SB, Connolly GN, Zhang L, Jia LT, Henningfield JE, Richter P. Global surveillance of oral tobacco products: total nicotine, unionised nicotine and tobacco-specific $\mathrm{N}$-nitrosamines. Tob Control. 2011 May;20(3): e2

17. National Cancer Institute and Centers for Disease Control and Prevention. Smokeless Tobacco and Public Health: A Global Perspective. Pag 89-90. Bethesda, MD: U.S. Department of Health and Human Services, Centers for Disease Control and Prevention and National Institutes of Health, National Cancer Institute. 2014; NIH Publication No. 14-7983. Disponible en: https://cancercontrol.cancer.gov/brp/tcrb/smokeless-tobacco. (Acceso 16 de marzo de 2020)

18. Dalberto D, Cardoso C, Hilario AL, Perachi A, Grivicich I, da Silva J. Cytotoxic and genotoxic evaluation of cotinine using human neuroblastoma cells (SH-SY5Y) Genetics and Molecular Biology.2020. 43, 2, e20190123.

19. Grizzell JA and Echeverria V.New insights into the mechanisms of action of cotinine and its distinctive effects from nicotine. Neurochem Res. 2015, 40:2032-2046.

20. WORLD HEALTH ORGANIZATION. IARC Monographs on the Evaluation of Carcinogenic Risks to Humans. VOLUME 89 Smokeless Tobacco and Some Tobacco-specific N-Nitrosamines, pag.57-60. WORLD HEALTHORGANIZATION, INTERNATIONAL AGENCY FOR RESEARCH ON CANCER, 2007. [consulta el 03 de marzo de 2021].

21. Granero R, Jarpa P. Uso de chimo entre adolescentes en Venezuela. Encuesta Mundial sobre Tabaquismo en Jóvenes 1999-2008. Acta Odontol Venezol. 2011;49(3):1-9. Spanish.

22. Granero R, Cabré $S$. Tendencias en el uso de tabaco y sus factores de riesgo entre estudiantes del septimo al noveno grado de la escuela básica: estado Lara (Venezuela). Encuesta Mundial sobre Tabaquismo en Jóvenes 2000, 2004, y 2008. Av Cardiol. 2010;30(4):309-315. Spanish.

23. De Salazar L. Caracterización del programa Escuelas Libres de Humo de Tabaco en el marco de la Ley Orgánica de Protección al Niño, Niña y Adolescente (LOPNA). In: de Salazar L. Efectividad en promoción de la salud y salud pública: reflexiones sobre la práctica en América Latina y propuestas de cambio. Cali, Colombia: Universidad del Valle; 2009, p. 348-9. Spanish.

24. Infante E, Navarro A, Finizola R, Zevallos J, Moya D, Alvarado $\mathrm{S}$, et al. Estudio de la salud cardiovascular en el estado Lara (escel 2008) metodología del proyecto y perfil de la muestra estudia. Av Cardiol. 2009;30(4):316-25. Spanish. 
25. Muthukrishnan A, Warnakulasuriya S. Oral health consequences of smokeless tobacco use. Indian J Med Res. 2018;148(1):35 $\square 40$.

26. WHO global report on trends in prevalence of tobacco smoking 2000-2025, third edition. Pag. 1. Geneva: World Health Organization; 2019. Disponible en: https://www.who.int/ publications/i/item/who-global-report-on-trends-in-prevalenceof-tobacco-use-2000-2025-third-edition. (Acceso 2 de abril de 2020) cia 14 páginas

27. WHO Report on the Global Tobacco Epidemic, 2019. Pag. 76 Geneva: World Health Organization; 2019. Disponible en: https://www.who.int/tobacco/global_report/en/. (Acceso 20 de marzo de 2020) cita 15 paginas

28. Organización Panamericana de la Salud. Informe sobre el control del tabaco en la Región de las Américas, 2018. Pag. 13 Disponible en: https://iris.paho.org/handle/10665.2/49237. (Acceso 15 de abril de 2020)

29. Organización Panamericana de la Salud. Informe sobre el Control del Tabaco en la Región de las Américas. A 10 años del Convenio Marco de la Organización Mundial de la Salud para el Control del Tabaco. Pag. 10 Washington, DC: OPS, 2016. Disponible en: https://iris.paho.org/handle/10665.2/28380. (Acceso 12 de enero de 2020)

30. Centers for Disease Control and Prevention. Global Youth Tobacco Surveillance, 2000-2007 MMWR. 2008. Pag. 2-3. Disponible en: https://www.cdc.gov/mmwR/pdf/ss/ss5701.pdf. (Acceso 11 de marzo de 2020)

31. Bermúdez J. El chimó, tabaco de mascar venezolano, cruza fronteras. Rev Venez Invest Odont IADR. 2021;9(1): 1-9.

32. Rostron BL, Chang JT, Anic GM, Tanwar M, Chang CM, Corey CG. Smokeless tobacco use and circulatory disease risk: a systematic review and meta-analysis. Open Heart. 2018 oct 8;5(2): e000846.

33. Sosa L, Rosales A, Dávila L, Quiñónez B, Jarpa P. Alteraciones histológicas ocasionadas por el tabaco de mascar venezolano (chimó) en los tejidos periodontales de ratas. Revista Cubana de Estomatología. 2009; 46(3): 38-47.

34. Rosales A, Sosa L, Dávila L, Quiñónez B, Jarpa P. Cambios clínicos periodontales ocasionados por el chimó en animales de experimentación. Revista Odontológica de los Andes. 2008 julio-diciembre; 3(2):21-27.

35. Álvarez M, Mujica S, Perdomo L, Navarro E. Extracto acuoso de tabaco masticable Chimó EI Tigrito $®$ y la disfunción cardíaca en modelo experimental larvario de pez cebra. Revista de Toxicología en línea (Retel). 2016 julio-septiembre; 47.

36. Febres N, Gilioli P, Olávez D, Omaña C, Solórzano E. Estudio in vitro de la erosión dental asociada al chimó. Univ Odontol. 2017 Ene-Jun; 36(76)

37. Jarpa P. Medición de $\mathrm{pH}$ de 12 preparaciones distintas de pasta de tabaco de mascar, relacionándola con la adicción a la nicotina. Revista de la facultad de Farmacia. 2003;45(2):7-11.

38. Millán-Mendoza B, Carrero S, Sánchez O, Urdaneta L. Caracterización microbiológica del chimó o tabaco de mascar consumido en el estado Mérida. Rev Fac Farm. 2009; 51 (2): 41-45.
39. Bermúdez, J. Lesiones en la mucosa bucal relacionadas con el consumo del tabaco de mascar (chimó). Reporte de un caso. Ciencia Odontológica. 2011 julio-diciembre; 8(2):141.

40. Parra J, Tovitto E, Jarpa P, Moreno G, Florido R, Omaña C. Determinación de cambios celulares en pacientes consumidores de chimó a través del estudio citológico. Rev Venez Invest Odont IADR 2014; 2 (2): 116-125.

41. Sosa M, Urdaneta L, Chidiak S, González A, Jarpa P Caracterización preliminar de la flora bacteriana en la biopelícula dental de individuos consumidores de chimó. Revista odontológica de Los Andes. Julio-diciembre 2008; 3 (2):4-12.

42. Sánchez N, Sosa M, Urdaneta L, Chidiak S, Jarpa P. Cambios en el flujo de $\mathrm{pH}$ salival de individuos consumidores de chimó. Revista odontológica de Los Andes. 2009 enero-junio; 4(1):613.

43. González-Rivas J, Nieto-Martínez R, García Santiago R, Ugel $\mathrm{E}$, Mechanick J. Chimo, a smokeless tobacco preparation, is associated with a higher frequency of type 2 diabetes. Invest. Clín. 2017;58 (3):250-258.

44. González-Rivas J, García Santiago R, Mechanick J, NietoMartínez R. O Chimó, uma Preparação de Tabaco sem Fumaça, está Associado a uma Frequência mais Baixa de Hipertensão em Indivíduos com Diabetes Tipo 2. International Journal of Cardiovascular Sciences. 2017;30(5)373-379.

45. Pozuelos E, Martinena E, Monago I, Viejo I, Pérez T. Farmacología de la nicotina. Medicina Integral, mayo 2000, 9(35):409-417.

46. Fernández E, Figueroa A. Tabaquismo y su relación con las enfermedades cardiovasculares. Rev Haban Cienc Méd. 2018 Abr; 17(2):225-235.

47. Messaouda O, Isabelle D, Mohamed L, Atika B, Abdelwahab $M$, Véronique L, Schüz J, Hocine B, Leon M. Smokeless Tobacco Use, Cigarette Smoking, and Upper Aerodigestive Tract Cancers: A Case-Control Study in the Batna Region, Algeria, 2008-2011. Tob Use Insights. 2020 febrero 5; 13: $1179173 \times 20902239$

48. Guha N, Warnakulasuriya S, Vlaanderen J, Straif K. Betel quid chewing and the risk of oral and oropharyngeal cancers: a meta-analysis with implications for cancer control. Int J Cancer. 2014; 135:1433-1443

49. Chaturvedi $P$, Singh A, Chien CY, Warnakulasuriya S. Tobacco related oral cancer. BMJ. 2019 junio 5; 365: 12142

50. Aguiar PH, Furtado NC, Repole BM, Ribeiro GA, Mendes IC, Peloso EF, Gadelha FR, Macedo AM, Franco GR, Pena SDJ. Oxidative stress and DNA lesions: The role of 8-oxoguanine lesions in Trypanossoma cruzi cell viability. PLoS Negl Trop Dis. 2013, 7:e2279.

51. Da Silva FR, Erdtmann B, Dalpiaz T, Nunes E, Ferraz A, Martins TLC, Dias JF, Da Rosa DP, Porawskie M, Bona S. Genotoxicity of Nicotiana tabacum leaves on Helix aspersa. Genet Mol Biol. 2013, 36:269-275.

52. Yildiz D. Nicotine, its metabolism and an overview of its biological effects. Toxicon, 2004, 43:619-632.

53. Van der Waal I. Oral potentially malignant disorders: is malignant transformation predictable and preventable? Med Oral Patol Oral Cir Bucal 2014;19(4): e386-390. 
54. Tanja AK, Quintero LCM. Efectos neurobioquímicos de la nicotina en el cerebro humano. 2015;54(260):31-41.

55. Pérez N, Pérez H, Fernández E. Nicotina y adicción: un enfoque molecular del tabaquismo. Rev haban cienc méd. 2007;6(1): ISSN 1729-519X

56. Rani A, Panchaksharappa M, Chandrashekarappa N, Annigeri $R$, Kanjani V. Characterization of saliva in immunocompromised patients and tobacco users: A case-control study.IJDR, 2019;30(6):909-914

57. Kanwar A, Sah K, Grover N, Chandra S, Singh RR. Long-term effect of tobacco on resting whole mouth salivary flow rate and $\mathrm{pH}$ : An institutional based comparative study. Eur J Gen Dent 2013; 2:296-299
58. Al-Ak'hali M, Halboub E, Mujam O, Alahmar $\mathrm{N}$, Jerah $\mathrm{S}$, Mutawwam F. Salivary parameters among Arabian snuff (Shammah) users. Saudi Med J. 2017 Dec;38(12):1201-1206.

59. Rooban T, Mishra G, Elizabeth J, Ranganathan K, Saraswathi TR. Effect of habitual arecanut chewing on resting whole mouth salivary flow rate and pH. Indian J Med Sci 2006; 60:95-105.

60. Albertson DG, Collins C, McCormick F, Gray JW. Chromosome aberrations in solid tumors. Nat Genet 2003, 34: 369-376.

61. Feltes BC, Poloni JF, Notari DL, Bonatto D. Toxicological effects of the different substances in tobacco smoke on human embryonic development by a systems chemo-biology approach. PLoS One. 2013; 8: e61743. 